\title{
La revolución epistemológica de las mujeres indígenas: una mirada a través de las propuestas de cuatro pensadoras contemporáneas
}

The epistemological revolution of indigenous women: a glimpse through the proposals of four contemporary women thinkers

A revolução epistemológica das mulheres indígenas: um olhar através das propostas de quatro mulheres pensadoras contemporâneas

Dora Arce-Rodríguez Universidad Nacional, Costa Rica Francisco Javier Rodríguez-Víquez

Universidad Nacional, Costa Rica

Mariana Castillo-Rojas

(ORCID: https://orcid.org/0000-0001-6210-2828)

Universidad Nacional, Costa Rica Recibido: 28/1/2021 • Aceptado: 3/3/2021

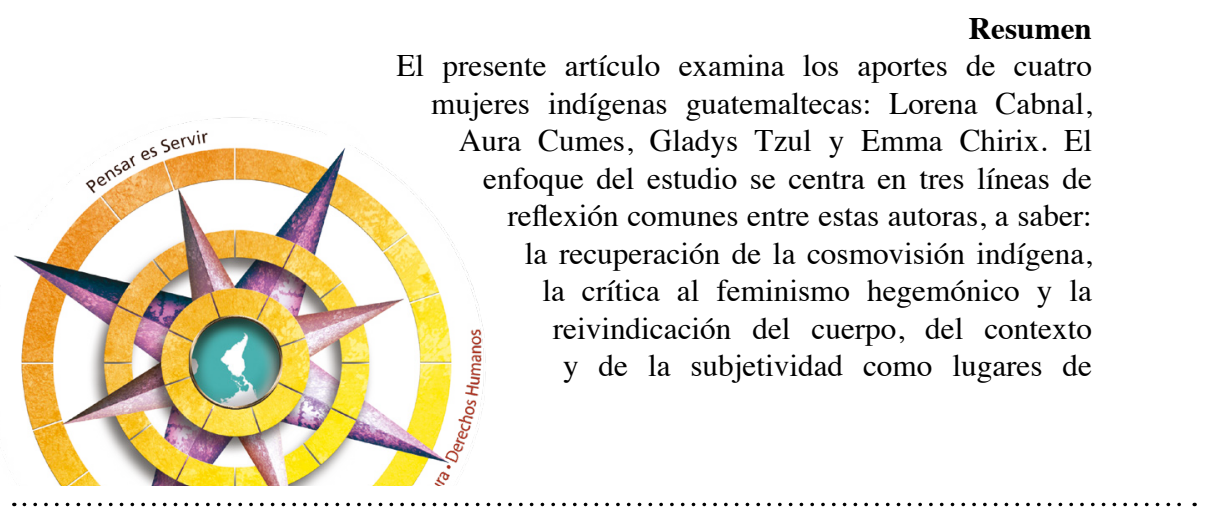


enunciación. A partir del diálogo entre sus propuestas, se evidencia el desarrollo de un corpus epistémico particular, centrado en la subjetividad y el contexto sociocultural e histórico de las mujeres como puntos de arranque para comprender la realidad.

Palabras clave: cosmovisión indígena, feminismos, corporalidad, Lorena Cabnal, Aura Cumes, Gladys Tzul, Emma Chirix.

\begin{abstract}
This article examines the contributions of four indigenous Guatemalan women: Lorena Cabnal, Aura Cumes, Gladys Tzul and Emma Chirix. The focus of the study is centered on three lines of reflection common to these authors, namely, the recovery of the indigenous cosmovision, the critique of hegemonic feminisms, and the vindication of the body, context and subjectivity as places of enunciation. From the dialogue between their proposals, it is evident the development of a particular epistemic corpus that focuses on subjectivity and the socio-cultural and historical context of women as starting points to understand reality.
\end{abstract}

Keywords: indigenous cosmovision, feminisms, corporeality, Lorena Cabnal, Aura Cumes, Gladys Tzul, Emma Chirix.

Resumo

Este artigo examina as contribuições de quatro mulheres indígenas guatemaltecas: Lorena Cabnal, Aura Cumes, Gladys Tzul e Emma Chirix. O foco do estudo centra-se em três linhas de reflexão comuns a estes autores, nomeadamente, a recuperação da cosmovisão indígena, a crítica do feminismo hegemónico, e a reivindicação do corpo, contexto e subjectividade como lugares de enunciação. Do diálogo entre as suas propostas, é evidente o desenvolvimento de um corpus epistémico particular que se concentra na subjectividade e no contexto sócio-cultural e histórico das mulheres como pontos de partida para a compreensão da realidade.

Palavras chave: cosmovisão indígena, feminismos, corporeidade, Lorena Cabnal, Aura Cumes, Gladys Tzul, Emma Chirix.

\section{Introducción}

Cinco siglos después de la invasión española a sus tierras, los conocimientos ancestrales de las comunidades indígenas perviven en medio de las constantes amenazas. La subsistencia de sus cosmovisiones es parte de la resistencia histórica de estos pueblos contra la imposición de la lógica de la modernidad occidental. A lo largo esta lucha, las mujeres indígenas han tenido un papel protagónico, aunque hayan sido doblemente invisibilizadas por el colonialismo y el

72 La revolución epistemológica de las mujeres indígenas: una mirada a través de las propuestas de cuatro pensadoras contemporáneas Dora Arce-Rodríguez, Francisco Javier Rodríguez-Víquez y Mariana Castillo-Rojas 
patriarcado. Hoy sus voces no solo denuncian la discriminación hacia sus poblaciones y las violencias particulares que sufren por su condición de género, clase y etnia, sino que también realizan importantes contribuciones al pensamiento decolonial latinoamericano.

El presente artículo examina los aportes del pensamiento de cuatro mujeres indígenas guatemaltecas: Lorena Cabnal, Aura Cumes, Gladys Tzul y Emma Chirix. Para ello, se enfocarán tres aspectos particulares abarcados por las autoras, que pueden considerarse preocupaciones comunes y, por ende, permiten un diálogo enriquecedor entre sus propuestas: la recuperación de la cosmovisión indígena, la crítica al feminismo hegemónico y la reivindicación del cuerpo, el contexto y la subjetividad como lugares de enunciación. Sus reflexiones han nutrido particularmente al feminismo decolonial y han colaborado con el desarrollo de un corpus epistémico particular que usa la subjetividad y el contexto sociohistórico de las mujeres como puntos de partida para comprender la realidad.

El posicionamiento epistemológico de estas autoras, con base en el diálogo entre sus propuestas, tiene una relevancia particular para el feminismo latinoamericano, por sus críticas al sesgo occidental que totaliza la experiencia de las mujeres de la región, anulando subjetividades e invisibilizando contextos específicos de opresión. Desde esa perspectiva, se trata de aportes imprescindibles para el campo de las epistemologías críticas feministas latinoamericanas.

\section{Recuperación de la cosmovi- sión indígena}

El entendimiento epistémico y ontológico de las autoras tiene como base su realidad ancestral, es decir, su lugar de enunciación es desde sus raíces. De esta forma, otorgan un valor fundamental a las sabidurías que comparten como pueblos indígenas, así como a las experiencias compartidas en el orden colonial. Al respecto, Gladys Tzul (2015a) señala lo siguiente:
Nuestras interpretaciones se construyen en espacios cotidia- nos y son el resultado de un lar- go proceso acumulativo de ex- periencias y estrategias políticas en el hacer de la vida. Cobran forma y fuerza desde el ejerci- cio de actualización de las ex- periencias políticas inspiradas en las rebeliones anticoloniales, y permanentemente disputan y construyen un orden simbólico.

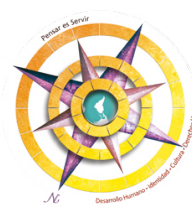


En suma, las comunidades han construido una inteligencia colectiva con capacidad de negociar y asediar a la dominación (p. 12).

De acuerdo con esta pensadora, las culturas indígenas han estado en constante amenaza por las lógicas coloniales que han desplazado sus conocimientos a una posición de poca o ninguna importancia, apostando por una ideología y una forma de existir individualista, en lugar de optar por un pensamiento y modo de vida colectivo. Frente a ello, estas comunidades "han construido sistemas de interpretación política; éstos han tenido efectos al interpelar y fracturar el orden de dominación y explotación colonial prolongada en la república y que hoy día da cuerpo al sistema político en Latinoamérica" (Tzul, 2015a, p. 12). De esta forma, su pensamiento se posiciona críticamente ante la historia que les ha sometido.

Por su parte, Emma Chirix destaca el papel del lenguaje en la transmisión de las visiones de mundo y, en particular, la centralidad de las lenguas indígenas en la reivindicación de esas formas de vida "otras" que han sido víctimas del etnocidio occidental. En su investigación, Chirix (2009) demuestra que en la concepción cultural maya tanto la sexualidad como la corporalidad eran entendidas en relación directa con la naturaleza. "El lenguaje hablado por la comunidad kaqchikel prioriza la colectividad. Asimismo, comunica los saberes sólo si están asociados a la posibilidad de la experiencia" (Chirix, 2009, p. 149).

La recuperación de los conocimientos ancestrales implica, a su vez, la crítica a esos valores occidentales impuestos sobre sus pueblos por el colonialismo. Esto ha dado paso a un profundo cuestionamiento de las estructuras sociales, particularmente, de las relaciones entre hombres y mujeres. La autora Aura Cumes ha realizado diversas investigaciones al respecto y ha llegado a conclusiones, las cuales ponen en evidencia que existen vínculos sociales distintos entre los sexos dentro de la cosmovisión maya:

En el caso de Guatemala, los pocos registros que existen muestran que los hombres no están en el centro de la conformación de la sociedad como raíz de pensamiento o epistemología, como sí ocurre en el pensamiento cristiano. Lo que dice el Popol Wuj, por ejemplo, es que cerca de once parejas formadoras son las que construyen -después de cuatro veces de ensayo - a la gente: cuatro hombres y cuatro mujeres. A partir de allí deciden crear a la

74 La revolución epistemológica de las mujeres indígenas: una mirada a través de las propuestas de cuatro pensadoras contemporáneas Dora Arce-Rodríguez, Francisco Javier Rodríguez-Víquez y Mariana Castillo-Rojas

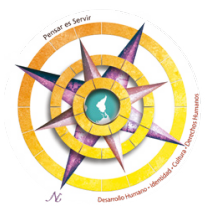


gente, y no se crea un hombre, se crean cuatro parejas (cuatro hombres primero y cuatro mujeres después). Además, el contenido de los idiomas mayas tiene relación con esto que está escrito en el Popol Wuj. En nuestras ceremonias - no politizadas - , la gente dice cada vez que invoca algo: matiox che qa tit qa mam ("gracias a nuestras abuelas-abuelos") (Yasic, 2014, párr. 45).

Como se puede observar, el análisis crítico que realizan estas autoras no se limita a cuestionar lo colonial, sino que también determina la existencia de marginación y discriminación en sus propias culturas. De allí que pensadoras como Lorena Cabnal también critiquen los aspectos machistas que pueden encontrarse en las poblaciones originarias de América. Para ella, en la cosmovisión indígena se han establecido patrones de conducta que indican cómo se debe comportar una mujer y un hombre a lo interno de la sociedad, limitando y oprimiendo, así, a las demás personas que no "caben" en estos dos grandes patrones. Como expresa Cabnal (2010), "este sistema establece su base de opresión desde su filosofía que norma la heterorrealidad cosmogónica como mandato, tanto para la vida de las mujeres y hombres y de estos en su relación con el cosmos" (p. 14).
No se trata entonces de una recuperación acrítica del pensamiento indígena, es, más bien, la apropiación de conocimientos históricamente marginados como parte de un posicionamiento teórico y político. $\mathrm{Al}$ respecto, son esclarecedoras las palabras de Tzul (2015a):

Nuestras interpretaciones se
construyen en espacios cotidianos
y son el resultado de un largo pro-
ceso acumulativo de experiencias
y estrategias políticas en el hacer
de la vida. Cobran forma y fuerza
desde el ejercicio de actualiza-
ción de las experiencias políticas
inspiradas en las rebeliones anti-
coloniales, y permanentemente
disputan y construyen un orden
simbólico (p. 12).

\section{Crítica al feminismo occidental hegemónico}

Partiendo de estas posiciones críticas que cuestionan los sistemas de opresión en los que han tenido que desarrollarse, desde su experiencia como mujeres indígenas, las autoras logran aportar una visión con otras aristas de análisis del pensamiento feminista. Su crítica del feminismo hegemónico radica, principalmente, en la universalización de la experiencia de las mujeres a través de la negación de la diversidad del concepto de "mujer", que invisibiliza 
los contextos y las muy diversas formas de violencia. $\mathrm{Al}$ respecto Cumes (2009) señala que "quizás el problema de estas vertientes del feminismo es creer que todas las mujeres tienen las mismas problemáticas, los mismos reclamos y que todas - como género - tienen una lucha exclusivamente contra el patriarcado" (p. 32).

En este respecto, y desde las epistemologías críticas feministas, Mohanty (2008) demostró, a partir de un estudio de diversas investigaciones académicas, que ha existido una tendencia del feminismo occidental a generalizar las experiencias de opresión de las mujeres de países considerados "Tercer Mundo". Esta problemática clasificación, que pone en evidencia, una vez más, el eurocentrismo de la modernidad, contribuye a invisibilizar las subjetividades y los contextos particulares de estas mujeres. Además, provoca que sean relegadas al lugar de "ser colonizado", víctima indefensa y sin voz. En la introducción de su texto Bajo los ojos de Occidente. Academia Feminista y discurso colonial, la autora deja claro ese posicionamiento:

Argumento aquí que las premisas de privilegio y universalismo etnocéntrico, por una parte, y la conciencia inadecuada de la academia occidental sobre el "tercer mundo", en el contexto de un sistema mundial dominado por Occidente, por la otra caracterizan una parte significativa de las obras feministas occidentales sobre las mujeres del tercer mundo. Un análisis de la "diferencia sexual" en forma de una noción monolítica, singular, y transcultural del patriarcado o la dominación masculina no puede sino llevarnos a la construcción de una noción igualmente reduccionista y homogénea de lo que yo llamo "la diferencia del tercer mundo" - ese concepto estable, antihistórico, que aparentemente oprime a la mayor parte, si no es que a todas las mujeres de todos los países (Mohanty, 2008, p. 116).

La cita anterior evidencia una grave problemática en el llamado "feminismo blanco" que, como producto de la misma modernidad, arrastra muchos de sus más grandes errores. Suponer y manifestar que pueda existir un mismo concepto que abarque a todas las mujeres del mundo, aparte de ser esencialista, es bastante limitado, pues termina convirtiéndose en otro sistema rígido y opresor. Ese feminismo excluyente de herencia colonial constituye un método más de imposición del pensamiento occidental sobre las poblaciones indígenas. En esta lógica se validan, principalmente, las epistemologías que han sido desarrolladas en la academia, expuestas por personas

76 La revolución epistemológica de las mujeres indígenas: una mirada a través de las propuestas de cuatro pensadoras contemporáneas Dora Arce-Rodríguez, Francisco Javier Rodríguez-Víquez y Mariana Castillo-Rojas 
mayormente blancas y con múltiples estudios universitarios.

En tal escenario de exclusión epistémica, los pareceres de las autoras, en particular de Cabnal y Chirix, reivindican el diálogo reflexivo e integrador entre los diversos contextos, posiciones y subjetividades de las mujeres. En este sentido, se recalca la importancia de construir a partir de la diferencia, para lograr articular estrategias comunes para el bienestar de todas:

Este hilo del pensamiento, de la palabra y de la acción feminista comunitaria me ha llevado a ver la importancia de tejer pensamientos con otras mujeres, sean indígenas de los diversos pueblos originarios, o sean "occidentales", porque creo que nos conviene a todas, propiciar espacios y encuentros para reflexionarnos, para atrevernos a hacer desmontajes y para construir en colectividad transgresión (Cabnal, 2010, p. 25).

Como respuesta a la mirada eurocéntrica colonial excluyente que socava los esfuerzos por articular propuestas de emancipación conjuntas, el sentido de comunidad, propio de la cosmovisión indígena, y el reconocimiento de las opresiones permiten un acercamiento integrador de las diversas construcciones de mundo.
Así, la comprensión de la complejidad humana, a partir del rechazo a la imposición del eurocentrismo totalizador, da paso al desarrollo de una propuesta epistemológica mucho más amplia, integradora y abierta al diálogo constructivo, en pro de la transformación social.

\section{Reivindicación del cuerpo, el contexto y la subjetividad como lugares de enunciación}

Sin duda, es necesario conocer los contextos para dilucidar las causas de los fenómenos. En ese sentido, las pensadoras indígenas realizan un aporte epistémico fundamental, al producir conocimiento desde sus propias realidades, desde sus cuerpos, desde la tierra misma que habitan y las habita. Ellas no temen hablar, levantar su voz desde donde se desarrollan sus íntimas vivencias y diversas cotidianidades. Es así como se reapropian de su subjetividad y la convierten en una forma de comprender su realidad.

El pensamiento de las mujeres indígenas busca un posicionamiento epistemológico crítico, ante violencias a las que han sido sometidas durante años. En ese sentido, $\mathrm{Cu}$ mes (2014) reconoce la importancia de que se escuchen las voces de esas féminas, como parte de la 
reestructuración social necesaria para mejorar las condiciones de vida de las comunidades:

La idea es permitir que estas prácticas de resistencia nos enseñen las interconexiones complejas de las estructuras de poder que, para el caso de Latinoamérica, sobrepasan la cuestión económica. Algunos análisis de izquierda se refieren al colonialismo solamente como un proyecto económico traído por los procesos de invasión, pero su permanencia muestra que se trata más bien de una imposición, además de económica y política, epistémica, en cuya base se estructuran las sociedades colonizadas (p. 67).

Otro aspecto importante de señalar acerca del posicionamiento epistemológico de estas mujeres indígenas, que a su vez está relacionado con la necesidad de conocer el contexto, es el de cómo abordan a sus sujetos de estudio. A diferencia de la mayoría de las prácticas academicistas que observan y analizan de manera descontextualizada, estas pensadoras reconocen el valor de comprender la vida, los cuerpos, contextos y territorios de las mujeres o comunidades que investigan desde cada uno de esos espacios, para hacerle frente a las múltiples formas de opresión que experimentan. Esto implica una ruptura con la idea de que se necesita distancia entre la persona investigadora y el "objeto de estudio"; al contrario, ellas abogan por establecer diálogos que permitan la contextualización y la comprensión profunda desde la subjetividad. De ahí que sea fundamental recuperar la voz de quienes son silenciadas.

Por las razones aludidas, los análisis de las estudiosas contemplan los diversos lugares de enunciación desde donde se produce el conocimiento y las relaciones que existen entre estos aspectos. Además, se posicionan a partir de su propia experiencia, es decir, ellas mismas forman parte viva de lo que investigan, así como de sus luchas. Sobre esta forma de construir epistemología, Cabnal (2010) se expresa de la siguiente manera:

El reto de repensarnos y de ir reconociéndonos en sujetos y sujetas con derecho epistémico para crear pensamiento propio y con ello ir estableciendo nuevos paradigmas que nos permitan trascender las opresiones y envolver a las otras y los otros en esta responsabilidad de transformación profunda que es corresponsabilidad de todas y todos, para promover la justicia, la equidad, paz y la vida en plenitud (p. 22)

La compresión de las formas particulares opresivas que impone sobre ellas su condición de mujeres

78 La revolución epistemológica de las mujeres indígenas: una mirada a través de las propuestas de cuatro pensadoras contemporáneas Dora Arce-Rodríguez, Francisco Javier Rodríguez-Víquez y Mariana Castillo-Rojas

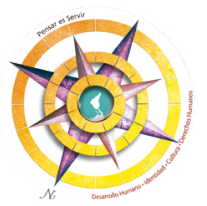


indígenas implica, para estas pensadoras, un lugar particular desde el cual pueden posicionarse para criticar la dominación colonial heteropatriarcal occidental. Según Tzul (2015b), esta apropiación del lugar enunciativo también es una respuesta contrahegemónica a la imposición cultural lingüística, pues "[aunque] muchas veces nuestras luchas contra los embates no las sentimos contenidas ni en los lenguajes ni en los actos más conocidos; esto no significó que olvidáramos la cuestión fundamental de nombrarnos como queríamos" (p. 99).

La reivindicación de su subjetividad, a partir del contexto y del cuerpo particularmente, implica, de igual forma, deconstrucción y reconstrucción de sí mismas. La reproducción de estereotipos relacionados tanto con su etnia como con su género y posición social (perpetuados por políticas de segregación, legitimadas y naturalizadas por la sociedad occidentalizada) las reprime de una manera especialmente recrudecida en su dimensión sexual y emocional.

Para Chirix (2014), "estas son construcciones sociales que nacen como una forma dominante de identidad en nuestra sociedad jerarquizada con la función de devaluar, someter y controlar" (p. 213). Esto es perpetuado por políticas excluyentes que se encuentran legitimadas y naturalizadas por la sociedad occidentalizada. Dicha visión eurocéntrica no solo discrimina a las poblaciones indígenas, sino que también justifica, a través de la dominación cultural, la explotación de sus cuerpos, saberes $\mathrm{y}$ territorios.

De esta forma, la revaloración de la identidad de las autoras y de su relación consigo mismas se vuelve, entonces, un acto político vinculado con la experiencia colectiva. Por lo tanto, se trata también de visibilizar el lugar preponderante que han tenido las mujeres dentro de sus comunidades. Para Tzul, es necesario que la transformación de las relaciones sociales se lleve a cabo en el nivel de los roles familiares, de manera que haya una distribución justa de las responsabilidades, la cual permita el bienestar integral de las mujeres. La idea es que estas puedan influir, más concretamente, en las decisiones políticas comunitarias y en la mejora de la calidad de vida de quienes integran la comunidad:

Si vivimos en relaciones sociales que producen comunidad, entonces tenemos que pensar con seriedad que debemos organizar y crear formas de responsabilidad y trabajo compartido entre mujeres y hombres, porque el cuidado no 
tiene que ser a costa de la salud de las mujeres. También tenemos que producir maneras en las que participemos plenamente no sólo en el uso de las tierras comunales, sino también en el proceso de la producción de la decisión sobre lo colectivo. Porque ahí se juega la permanencia de nuestra existencia a largo plazo (Tzul, 2015b, p. 99).

Por su parte, Chirix habla de la necesidad de superar el discurso colonial, para recuperar visiones de mundo que pongan la atención en la subjetividad de las personas y les permitan relacionarse de maneras más amorosas y solidarias. En ese sentido, la autora se posiciona desde una perspectiva crítica de los derechos humanos, que no solo rechaza la mirada occidental eurocéntrica, además coloca a las personas como agentes de lucha y reivindicación social. Debido a lo anterior, la estudiosa afirma que es imprescindible apropiarse de la subjetividad más allá del individualismo inculcado por la modernidad occidental, para comprenderse como parte de un tejido social que conforma la colectividad:

La subjetividad no trata solo lo personal sino lo social, es saber y sentir quién soy y lo que existe a mi alrededor; es conocer el proceso histórico, identificar las políticas de segregación, asimilación y etnocidio que el Estado, los grupos de poder económico y sectores ladinos siguen promoviendo para mantener sus privilegios $\mathrm{y}$ marcar siempre las desigualdades. La subjetividad invita a interpretar y no a naturalizar nuestra vida y nuestra identidad, a partir de raíces históricas, económicas, políticas y culturales para poder dar respuesta a las distintas causas de la opresión" (Chirix, 2014, p. 211).

En ese sentido, se da paso a la corresponsabilidad que vincula a los seres humanos con su comunidad y permite la construcción de sociedades en las que el bienestar común tiene un papel preponderante. Se trata, por tanto, de un posicionamiento que cuestiona directamente las dicotomías individual/social, modernidad/ barbarie, cuerpo/mente, etc., a partir de la comprensión contextualizada de la realidad y de nuevas relaciones inter- e intrapersonales.

\section{Reflexiones finales}

El análisis de los posicionamientos de estas cuatro autoras, sin lugar a dudas, pone de manifiesto la resistencia epistemológica de las mujeres y de los pueblos indígenas en América Latina. Si bien estas pensadoras no necesariamente se reconocen como feministas, sus propuestas se

80 La revolución epistemológica de las mujeres indígenas: una mirada a través de las propuestas de cuatro pensadoras contemporáneas Dora Arce-Rodríguez, Francisco Javier Rodríguez-Víquez y Mariana Castillo-Rojas

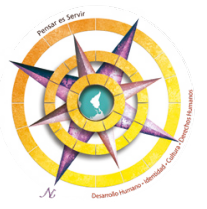


encuentran muy alineadas con el pensamiento crítico y decolonial feminista desarrollado en las últimas décadas en el Sur Global.

Es interesante observar cómo las demandas de autonomía intelectual para pensar(se) y repensar(se) desde la fuerte crítica al feminismo occidental están presentes en los movimientos de mujeres en todo el mundo. Esta es, indudablemente, una de sus principales estrategias, con el afán de apropiarse de su capacidad de producir conocimiento, como estrategia contrahegemónica ante los diversos sistemas de opresión que condicionan su vida y restringen su aptitud de incidencia en la propia realidad.

En ese sentido, se hace necesario destacar el potencial transformador que tienen estas aproximaciones críticas para el movimiento y la teoría feministas. Los problemas de articulación entre las agrupaciones sociales y las dificultades que instan llevar a cabo estrategias de acción política a mediano y largo plazo, en defensa de los derechos de las mujeres, están fuertemente vinculados con el origen liberal del feminismo. La falta de horizontalidad, la invisibilización de factores como el colonialismo, el racismo y el clasismo, al igual que el establecimiento de agendas de trabajo que no toman en cuenta la diversidad de voces para integrarlas en las mesas de diálogo constituyen algunos de los aspectos que alejan a muchas mujeres de estos espacios.

La comprensión de la diversidad humana y, particularmente, de las identidades culturales, subjetividades y contextos particulares es indispensable para tejer redes de apoyo entre mujeres. No se trata solamente de pensar en incidencia política o acción social, sino también de crear espacios seguros donde desarrollar relaciones afectivas que contribuyan al bienestar emocional de todas ellas. Cabe recordar que, para que puedan ejecutarse estrategias conjuntas, es necesaria no solo una buena coordinación basada en comunicarse efectivamente; además, se requiere confianza, compañerismo y sororidad/solidaridad.

La opresión patriarcal que compartimos puede ser nuestro lugar de encuentro para que, con base en nuestras experiencias particulares, podamos construir propuestas integrales más efectivas contra las distintas violencias que sufrimos las mujeres en nuestros contextos específicos. La lucha por nuestra dignidad humana requiere esfuerzos conjuntos, pues implica una deconstrucción de las imposiciones de la modernidad occidental, que dé paso 
a la transformación social y la configuración de relaciones sociales más justas e igualitarias.

Leer a estas autoras implica conocer su visión de mundo, así como el modo por medio del cual son confrontadas con nuestra propia concepción de la realidad y nuestra relación con nosotras mismas. Ya sea a partir del cuestionamiento a la individualidad androcéntrica, al patriarcado cristiano que nos priva del placer de nuestros propios cuerpos, etc., las propuestas de las estudiosas nombradas permiten que nos miremos desde sus posturas epistemológicas y que, por tanto, nos hagamos preguntas que nos pueden resultar incómodas y a la vez transformadoras.

Como personas investigadoras somos enfrentadas a otras visiones de mundo, a otras realidades que quizás desconocíamos o de las que sabíamos muy poco. Esto conlleva tanto un reto intelectual como una decisión ética, en relación con nuestra propia práctica epistemológica. Tenemos, por tanto, la responsabilidad de repensarnos y reconstruirnos a la luz de conocimientos situados, es decir, en vínculo con aspectos concretos de la vida de las mujeres. La opresión no es algo abstracto y, en consecuencia, nuestra aproximación a ella no puede ser si no ocurre a partir de la subjetividad y el contexto particular donde ocurre.

\section{Referencias bibliográficas}

Cabnal, L. (2010). Feminismos diversos: "El feminismo comunitario". Madrid: ACSUR-Las Segovias.

Chirix, E. (2009). Los cuerpos y las mujeres kaqchikeles. Desacatos, (30), 149-160.

Chirix, E. (2014). Subjetividad y racismo: la mirada de los otros y sus efectos. En Y. E. Miñoso, D. G. Correal y K. O. Muñoz (Eds.), Tejiendo de otro modo: Feminismo, epistemología y apuestas descoloniales en Abya Yala. Colombia: Universidad del Cauca.

Cumes, A. (2009). Multiculturalismo, género y feminismos: mujeres diversas, luchas complejas. En A. Pequeño (Comp.), Participación y políticas de mujeres indígenas en contextos latinoamericanos recientes (pp. 29-53). Quito: FLACSO Ecuador / Ministerio de Cultura.

Cumes, A. (2014). Esencialismos estratégicos y discursos de descolonización. En M. Millán, Más allá del feminismo: caminos para andar (61-86.). México: UNAM, Universidad Nacional Autónoma de México.

Mohanty, C. (2008). Bajo los ojos de Occidente. Academia Femista y discursos coloniales. En L. Suárez y A. Navaz (Eds.), Descolonizando el feminismo. Teorías y Prácticas desde los Márgenes. Valencia: Universidad de Valencia.

82 La revolución epistemológica de las mujeres indígenas: una mirada a través de las propuestas de cuatro pensadoras contemporáneas Dora Arce-Rodríguez, Francisco Javier Rodríguez-Víquez y Mariana Castillo-Rojas

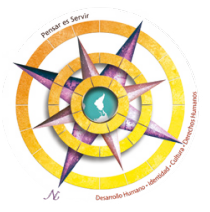


Tzul, G. (2015a). ¿Cómo construyen crítica las comunidades indígenas? Un acercamiento a las formas de la exclusión epistémica. Lasaforum Review, $X L V I(1), 12-13$.

Tzul, G. (2015b). Mujeres indígenas: Historias de la reproducción de la vida en Guatemala. Una reflexión a partir de la visita de Silvia Federici. Bajo el volcán, 15(22), 91-99.

Yaksic, M. (2014). Aura Cumes: "Tenemos que sacudirnos las telarañas del pensamiento único que encubren el despojo". Recuperado de https:// latinta.com.ar/2017/04/aura-cumes-tenemos-que-sacudirnos-las-telaranas-del-pensamiento-unico-que-encubren-el-despojo/ 
